\title{
AUTOMAÇÃO DE LABORATÓRIO DE ANÁLISES DE AMOSTRAS DE MINÉRIO DE FERRO
}

\author{
Fernando de Souza Paiva ${ }^{1,2}$ \\ Gabriel Duarte Lott ${ }^{2,3}$ \\ Gleison de Oliveira Duarte (3,4 $^{2,3}$ \\ Mario Alzamora Gomes ${ }^{2,3}$ \\ Rodrigo Alves Costa ${ }^{2,3}$
}

\begin{abstract}
Resumo
O processo de análises físicas e químicas de amostras de minério de ferro é um processo crucial para avaliação do tipo e da qualidade do minério que está sendo extraído e beneficiado nas operações das mineradoras. Aplicar novos conceitos e equipamentos altamente tecnológicos, que irão garantir resultados cada vez mais precisos e a execução das tarefas de uma forma cada vez mais segura, é a chave e o desejo dessas empresas. No laboratório da VALE S/A, em Itabira, implementou-se um sistema automatizado de preparação de amostras capaz de realizar as etapas de divisão da amostra abaixo de Imm, pulverização, confecção de pastilha prensada e fundida, análise de perda por calcinação (PPC), limpeza de utensílios e acessórios e descarte de resíduo de minério de ferro. Aborda-se nesse estudo uma avaliação dos equipamentos e tecnologias instalados e os desafios tecnológicos e de processos enfrentados durante a implantação desse projeto.

Palavras-chave: Automação de processos; Laboratório; Integração de sistemas.
\end{abstract}

\section{AUTOMATION OF LABORATORY OF IRON ORE SAMPLES ANALYSIS}

\begin{abstract}
The process of physical and chemical analysis of iron ore samples [I] is a crucial process for evaluating the type and the quality of ore that is being mined and benefited in mining operations. Applying new concepts and highly technological equipment, which will ensure increasingly accurate results and the execution of tasks in an increasingly safe way, is the key and the desire of these companies. In the laboratory of VALE S/A, in Itabira, an automated sample preparation system was implemented, which is capable of perform the steps of dividing the sample below $1 \mathrm{~mm}$, spraying, confection of pressed pellets and fused beads, analysis of loss of calcination (PPC), cleaning and disposal of iron ore residue. This study reports the evaluation of installed equipment and technologies and the technological and process challenges faced during the implementation of this project.
\end{abstract}

Keywords: Process automation; Laboratory; Integration of automation systems.

\section{INTRODUÇÃO}

O minério de ferro é um insumo essencial na sociedade da forma como está estabelecida atualmente. E a medida em que as sociedades se desenvolvem e evoluem, maior é a voracidacde das populações para obtenção e utilização desses minerais. Em contrapartida a dependência e maior demanda e utilização do minério de ferro e seus derivados, 0 aço principalmente, há uma crescente pressão nas empresas visando aumento de produtividade e redução de custos.
Dessa forma, os profissionais das empresas mineradoras estão sendo desafiados a aperfeiçoar os processos existentes, além de projetar as novas instalações para trabalharem com altos níveis de eficiência [I].

Garantir uniformidade e qualidade em cada etapa [2] é um desafio e um desejo das equipes responsáveis pelos laboratórios de preparação e análises de amostras. Nesse contexto está inserido o laboratório da Vale em Itabira / MG,

'Centro Universitário do Leste de Minas Gerais, Ipatinga, MG, Brasil.

${ }^{2}$ VALE S/A, Itabira, MG, Brasil. E-mail: gabriel.lott@vale.com

${ }^{3}$ Universidade Federal de Minas Gerais, Belo Horizonte, MG, Brasil.

${ }^{4}$ Pontifícia Universidade Católica (PUC), Belo Horizonte, MG, Brasil.

2176-1523 (C) 2019 Associação Brasileira de Metalurgia, Materiais e Mineração. Publicado pela ABM. Este é um artigo de acesso aberto distribuído sob os termos da licença Creative Commons CC BY-NC-ND (Attribution-NonCommercial-NoDerivs) - https:// creativecommons.org/licenses/by-nc-nd/4.0\%. 
que é caracterizado como um laboratório operacional, cuja função é apoiar o processo produtivo de diversos setores da cadeia de produção, desde as frentes de lavras das minas até o embarque do minério [3]. A Figura I apresenta o percentual de amostras por origem no laboratório de Itabira.

O processo de preparação e análise das amostras, contudo, desde o início das operações do laboratório de Cauê da Vale S/A., em Itabira, foi realizado com maquinário robusto, Figura 2, mas que exigiam a intervenção manual dos operadores [4].

Realizar uma transformação nessa forma de trabalho [5], com foco em automação e melhoria das condições de segurança para as pessoas, foi um dos motivadores para o projeto de modernização do laboratório, cujas etapas de divisão, moagem, fusão e análise das amostras pelos equipamentos de raios- $x$ foram automatizadas, visando aumentar a confiabilidade e reprodutibilidade dos procedimentos de preparação, garantindo uma maior qualidade das análises realizadas. Dessa forma, consegue-se gerar uma informação útil para que as áreas operacionais consigam ajustar as variáveis de processo de forma a garantir um produto dentro das especificações [6]. A Figura 3 apresenta um fluxograma simplificado do laboratório de Itabira, em destaque para a área hachurada em azul com as etapas que foram automatizadas.

O projeto implementado consiste de um sistema automatizado de preparação e análise de amostras composto de uma planta com quatro células robóticas e mais de 20 equipamentos, como moinhos de pulverização e

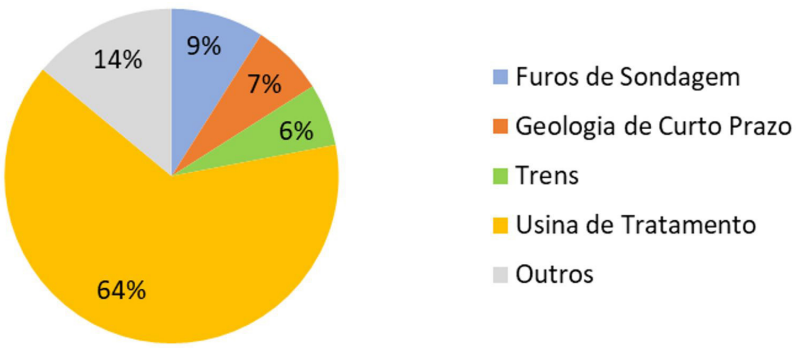

Figura I. Percentual (\%) de amostras por fonte de origem - Laboratório de Itabira.

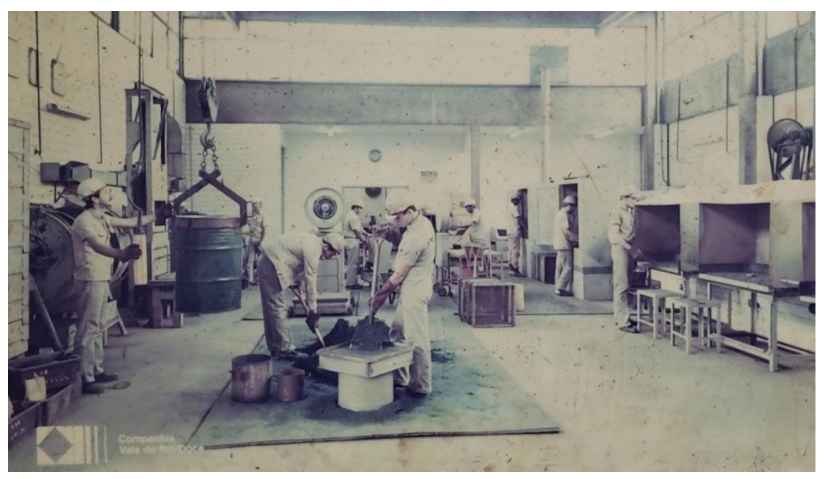

Figura 2. Início da operação do laboratório da Vale em Itabira / MG. prensagem, divisor de amostras, máquinas de fusão, esteiras transportadoras e robôs, numa área de aproximadamente $250 \mathrm{~m}^{2}$ e uma capacidade nominal de $\mathrm{I} 400$ amostras por dia.

A Figura 4 apresenta uma representação 3D do laboratório automatizado, com o destaque para os robôs localizados em cada uma das quatro células da planta.

Todos esses equipamentos possuem um controlador lógico programável (CLP) já utilizando tecnologia softPLC, arquitetura inovadora para aplicações que exigem alto desempenho de processamento, redes de comunicação internas de alta velocidade e tecnologia de ponta, tais como a rede de campo EtherCat, e ambientes computacionais virtualizados e redundantes, que facilitam a gestão da amostra desde $\mathrm{o}$ instante inicial de introdução na planta automatizada até o momento final da análise da composição química pelos espectrômetros de raios- $X$.

No entanto, a operação da planta é bastante simples, dada a complexidade e quantidade de equipamentos envolvidos: um laboratorista cadastra a amostra no sistema de entradas e alimenta uma das células robotizadas. A partir deste ponto, os robôs de cada uma das células são os responsáveis por conduzir a amostra pelos equipamentos da planta até a obtenção do resultado final da análise química e disponibilização dos resultados para os clientes do laboratório. Essa rota leva em torno de 10 minutos para ser completada, tudo feito de forma automática, sem intervenção direta do operador, cuja responsabilidade é fazer a supervisão remota de todo o processo via sala de operação e análise criteriosa dos resultados das análises das amostras.

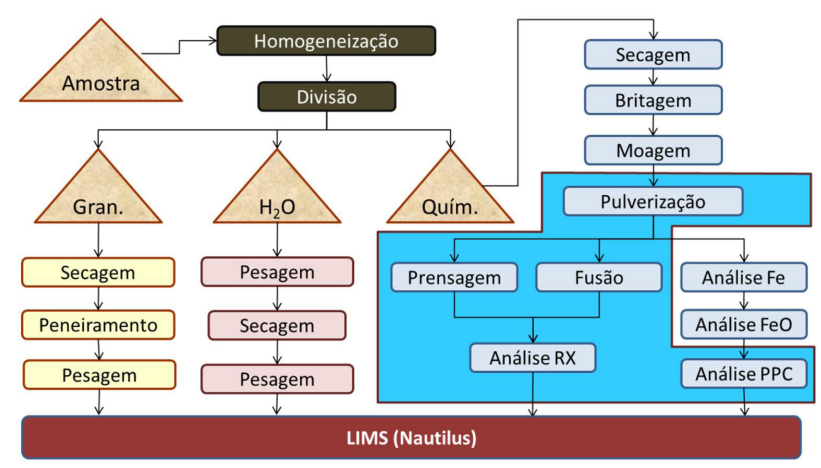

Figura 3. Fluxo de Processo - Laboratório de Itabira.

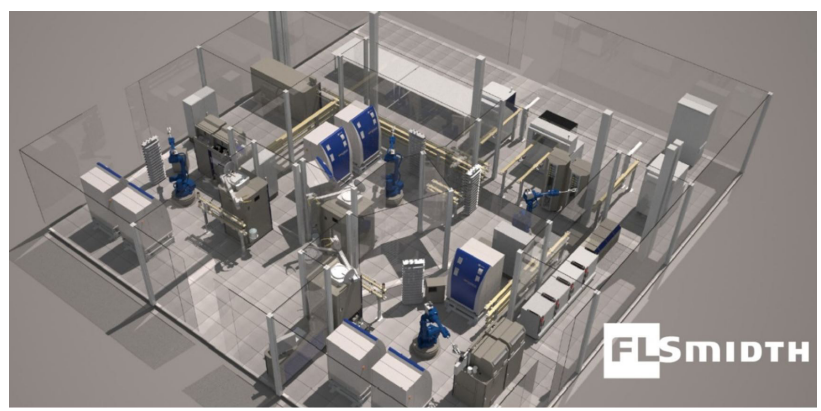

Figura 4. Planta Automatizada - Laboratório de Itabira. 


\section{METODOLOGIA}

Este trabalho é caracterizado como uma pesquisa descritiva, baseada em um estudo de caso realizado durante a implantação do projeto de automação do laboratório de análises físicas e químicas de amostras de minério de ferro da Vale S/A em Itabira.

O desenvolvimento desse projeto pioneiro na América Latina para análise química em laboratórios de minério de ferro foi iniciado por um longo trabalho de pesquisa e busca de soluções de automação de laboratório em outros países cuja tecnologia já se apresentava mais madura e robusta para esse tipo de aplicação. Baseado nas informações coletadas e com o know-how das equipes de automação e laboratório da Vale em relação ao processo e as tecnologias disponíveis no mercado, foi elaborada uma definição criteriosa das premissas, requisitos técnicos e características de uma solução de automação customizada para as necessidades e realidades do laboratório de Itabira.

A maturidade da solução foi obtida por meio de diversas reuniões de alinhamento técnico entre as partes envolvidas, como equipes de automação e laboratório da Vale, o fornecedor da solução de automação escolhido e fabricantes e fornecedores de equipamentos que foram integrados ao projeto. Um teste em fábrica em Wuppertal, na Alemanha, com o laboratório montado em escala real, foi determinante para avaliar a aderência da solução às necessidades da dinâmica exigida de uma operação 24 horas de um laboratório operacional, cujo prazo de resposta das análises é um fator determinante para a tomada de decisão dos clientes internos dessas informações, Figura $I$. A customização final foi garantida com o acompanhamento técnico das equipes de automação e laboratório da Vale e o fornecedor do projeto em campo, avaliando e operando a planta automatizada e tratando falhas observadas durante a fase de operação assistida.

\section{RESULTADOS E DISCUSSÃO}

O projeto de automação do laboratório tem como objetivo automatizar o processo de divisão da amostra, pulverização, confecção de pastilha prensada e pastilha fundida, análise de perda por calcinação (PPC) e análise por fluorescência de raios- $X$, de modo a se atingir os benefícios esperados, que são:

- Aumentar a eficiência e capacidade produtiva do laboratório, cuja planta automatizada tem capacidade nominal de análise de 1400 amostras por dia;

- Melhorar o atendimento aos requisitos de Saúde e Segurança Operacional (SSO) e atender às normas NR- 17 - Ergonomia com a redução da necessidade de transferência de amostras entre diversos equipamentos, uma vez que esse transporte na planta automatizada será executado internamente nos equipamentos ou por meio de robôs, não necessitando de intervenção do operador;

- Aumentar a confiabilidade e reprodutibilidade dos procedimentos e adequar-se aos procedimentos de preparação de amostras como os descritos na norma ISO 3082 (Iron ores - Sampling and sample preparation). Com a implantação do sistema automático, pretende-se garantir uma maior aderência as diretrizes da norma, agregando mais qualidade e representatividade aos procedimentos realizados;

- Garantir uma modernização e atualização tecnológica de todo o processo de preparação e análises químicas de amostras de minério de ferro do laboratório de Itabira, com a introdução de equipamentos novos e soluções inovadoras.

\section{I Infraestrutura de Rede e de Automação}

Para a integração de todos os equipamentos, fez-se necessário a definição de uma infraestrutura de comunicação de dados em rede de controle Ethernet-IP de alta capacidade de processamento e redundante, que permite a comunicação dos dados de todos os PLC's e remotas com os servidores. São dois servidores físicos distintos que fazem a comunicação com o campo também de forma independente, tanto lógica quanto fisicamente (há switches e cabos de rede específicos para cada um dos servidores), conforme Figura 5. Dessa forma, é garantida uma alta disponibilidade das aplicações da planta automatizada e da gestão das amostras dentro do sistema.

\subsection{Integração com Equipamentos e Sistemas já Existentes na Unidade}

A interface com outros sistemas já difundidos e maduros dentro da Vale, como os equipamentos de raios-x e o sistema de gerenciamento de resultados do laboratório, o Nautilus, de forma direta e automática, foi outro diferencial tecnológico propiciado pelo projeto. A análise das amostras pelos equipamentos de raios- $x$ e a transferência dos resultados para o Nautilus era realizada de forma manual, o que demandava dedicação e tempo para a realização de tal atividade.

O controle e operação dos equipamentos de raios- $x$ de forma automática pela planta automatizada, requisito do projeto, foi garantido por meio de desenvolvimento de uma solução integrada entre as equipes da Vale, o fornecedor do equipamento de raios- $x$ e o fornecedor da planta automatizada. Desenvolvimento de drivers de comunicação e controle específicos para o projeto precisaram ser elaborados, desenvolvidos e testados de forma conjunta, garantindo robustez e eficácia da solução e fácil intercambiabilidade entre a operação automático / manual do equipamento.

Tecnol. Metal. Mater. Miner., São Paulo, 


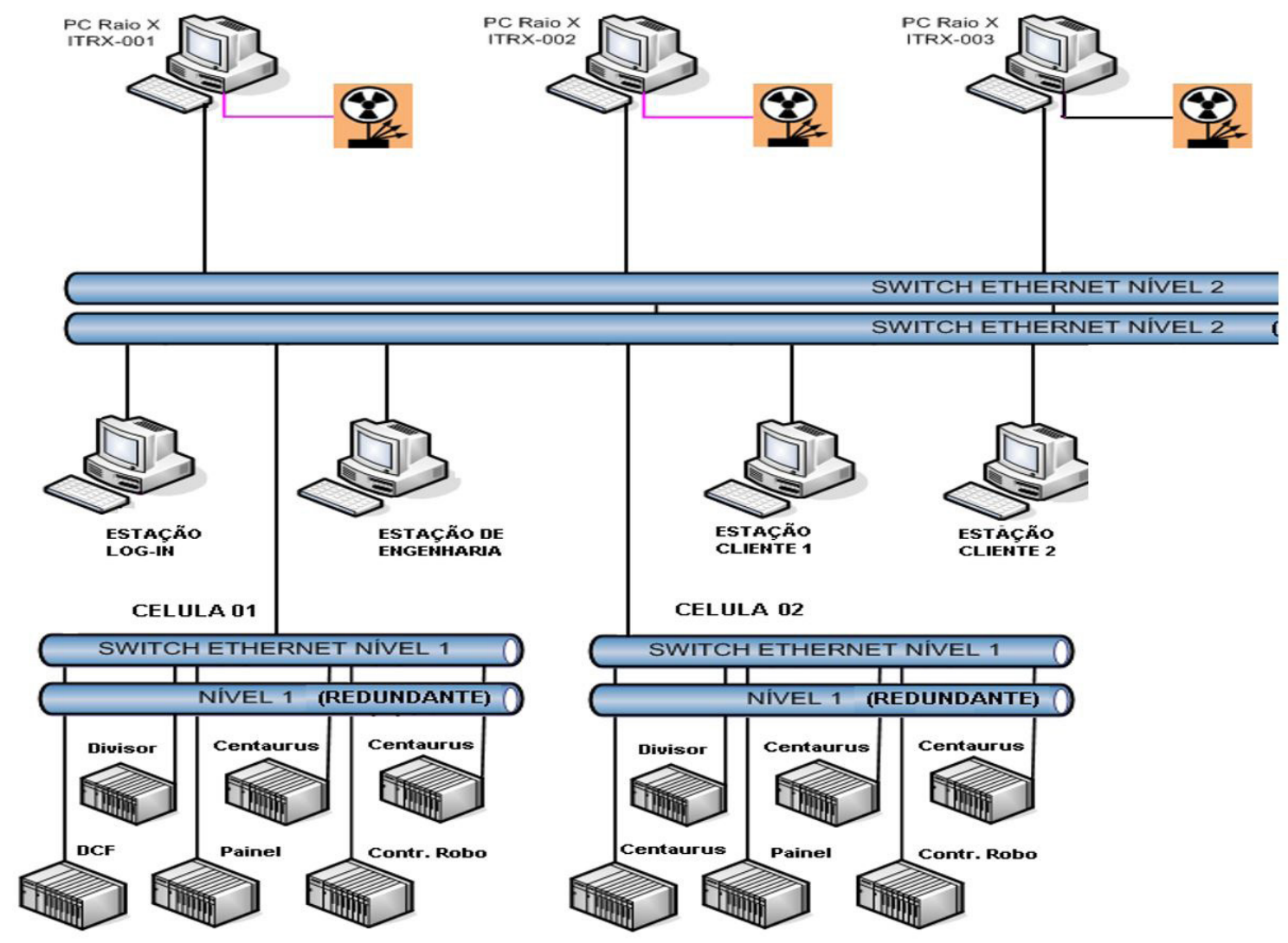

Figura 5. Infraestrutura de rede e automação - Planta automatizada.

Garantida a operação automática dos equipamentos de raios-X, o segundo grande desenvolvimento feito foi o envio automático dos resultados das amostras para o Nautilus, que tem como função gerenciar e garantir a confiabilidade e rastreabilidade de todos as informações das análises físico químicas das amostras processadas no laboratório, além de prover relatórios gerenciais e diversos indicadores do processo. Esta integração foi dividida em duas frentes. Na primeira, desenvolveu-se e implementou-se uma arquitetura de conexão segura, configurações e aplicações específicas, para permitir a comunicação entre sistemas alocados em redes de dados diferentes, sendo a planta automatizada na rede de dados industriais (automação) e o Nautilus na rede corporativa $(\mathrm{TI})$. Clientes webservices foram desenvolvidas para fazer o envio / recebimento de dados entre as diferentes interfaces de comunicação existente entre o servidor do Nautilus e o servidor da planta automatizada. No segundo momento, foi necessário todo um trabalho de padronização da estrutura dos dados, tanto das informações enviadas pelo Nautilus para fazer o registro inicial da amostra e sua rota de processo na planta, quanto do resultado das análises das amostras enviados pelos equipamentos. A partir de então, pode-se desenvolver as rotas de processos e parametrizações de cada um dos equipamentos para cada família de amostras de minério analisadas no laboratório, sempre buscando garantir uma boa reprodutibilidade e precisão dos resultados, de forma a produzir informações corretas aos clientes para a tomada de decisão.

\subsection{Inovação Tecnológica}

O Projeto de automação do laboratório de Itabira é o primeiro projeto de automação de laboratório da Vale para análise química, na qual vários processos (moagem, prensagem, fusão de amostras, envio de resultados para sistema de gestão, limpeza de copos e anéis de amostras) são realizados agora sem intervenção direta dos operadores, que fazem a leitura do código da amostra e inserção das amostras nas esteiras de entrada. Todo o restante do processo, como direcionamento das amostras dentro do sistema, rastreio de informações, interface com outros sistemas, movimentação da amostra entre os equipamentos, é toda feita pelo sistema de controle da planta automatizada, que é suportada por redes de comunicação de alta velocidade e performance, servidores físicos de última geração e ambiente virtualizado, equipamentos com PLC embarcado de alta capacidade de processamento.

O técnico será responsável por toda a gestão da planta automatizada via sala de controle, através de um sistema de supervisão que permitirá a interface dos operadores com o sistema, permitindo a visualização das variáveis e comandos dos equipamentos existentes, o que representa outro 
diferencial do projeto para a operação dos laboratórios da Vale. Esse profissional agora tem como função fazer a gestão das amostras na planta automatizada e garantir a qualidade dos resultados das análises das amostras de minério, ao invés de terem como rotina a inserção manual e rotineira de cada uma das amostras nos equipamentos de raios-x. Isso irá permitir ao técnico dedicar um tempo maior em atividades de maior exigência técnica, como análise qualitativa das amostras, uma vez que o cadastro e leitura manual das mesmas, que demandavam tempo, agora são controladas e executadas pela própria planta automatizada. Treinamentos específicos foram necessários para capacitar os operadores nesse novo modelo de trabalho, completamente diferente do método de trabalho vigente até então.

A utilização de ar comprimido e sistema de despoeiramento acoplado aos equipamentos foi outro fator que proporcionou vantagens e ganhos ambientais, ergonômicos e de produtividade para a operação do laboratório. $O$ próprio sistema gerencia e coordena 0 processo de limpeza dos copos e anéis de amostras e dos compartimentos dos equipamentos, com a utilização de ar comprimido. Tal processo anteriormente era feito manualmente pelos operadores, com a utilização de água e outros produtos, além de utilização de recipientes descartáveis para a produção dos anéis de amostras a serem analisados. Com isso, foi possível uma redução no consumo de água para a limpeza de equipamentos e redução de resíduos sólidos, além de ganhos de segurança ao minimizar a exposição ao risco nas operações rotineiras do laboratório.

\subsection{Testes de Performance e Qualidade dos Resultados}

De forma a mensurar a qualidade da solução proposta e $o$ atendimento dos requisitos definidos na etapa de especificação técnica, foram definidos testes de performance dos equipamentos de modo a garantir resultados das análises do minério de ferro com precisão e exatidão. Índices como disponibilidade física (DF) do sistema, contaminação cruzada entre amostras de teores químicos bastante diferentes, precisão e reprodutibilidade dos resultados, foram discutidos e executados durante a etapa de comissionamento do projeto. $O$ atendimento a esses requisitos, apesar de não garantir uma integralidade de conformidade da solução com todos os requisitos desejados, foram fundamentais para dar subsídios e comprovação técnica da qualidade dos resultados das amostras de minério de ferro produzidas pela planta automatizada baseadas em métodos consagrados pela literatura e conhecimento dos profissionais da área de processos de laboratório.

\subsection{Manutenção}

Capacitação técnica de toda a equipe de manutenção e automação da planta automatizada, que provê diagnósticos e alarmes na estação de engenharia e nos sistemas supervisórios e nas interfaces de operação local. Há a presença de mais de 2000 pontos de l/O's nos diversos equipamentos, o que garante maiores informações na identificação e tratativa das falhas. Um kit de peças sobressalentes, organizado e identificado em local adequado, garante uma maior produtividade da equipe de manutenção e um menor tempo de resolução das falhas, impactando menos possível a rotina de operação do laboratório.

\section{CONCLUSÃO}

O projeto foi desenvolvido de forma integrada entre as áreas de laboratório e de automação, que se uniram no desafio de modernizar e automatizar parte do processo de preparação e análise química do laboratório de Itabira. A implantação do projeto proporcionou:

- Aumento da eficiência e capacidade produtiva do laboratório;

- Melhoria do atendimento aos requisitos de Saúde e Segurança Operacional (SSO) e as normas NR-17 - Ergonomia;

- Modernização e atualização tecnológica;

- Redução na exposição ao risco nas tarefas de pulverização, prensagem, fusão de amostras e limpeza;

- Integração automática das informações com o Nautilus;

- Redução no consumo de água para a limpeza de equipamentos e redução de resíduos sólidos;

Além disso, o projeto implantado possui grande capacidade de expansão e aplicabilidade em outros laboratórios da empresa.

\section{REFERÊNCIAS}

I Benvindo da Luz, A.; Sampaio, J. A.; Matos de Almeida, S. L. Tratamento de minérios. 4a ed. Rio de Janeiro: CETEM; 2004.

2 Mazzinghy DB. Modelagem e simulação de circuito de moagem através da determinação dos parâmetros de quebra e da energia específica de fragmentação [dissertation]. Belo Horizonte: Universidade Federal de Minas Gerais; 2009.

3 Valadão, G. E. S., Viana, P. R. M., Araújo, A. C. Tratamento de minérios. 5a ed. Rio de Janeiro: CETEM; 2010.963 p.

4 VALE S.A. Vale: nossa história. I. ed. Rio de Janeiro: Verso Brasil Editora; 2012. v. I. 
Paiva et al.

5 Vale@informar - http://www.vale.com/brasil/pt/aboutvale/news/paginas/vale-inaugura-primeiro-laboratorioautomatizado-analise-de-quimica-minerio-ferro.aspx

6 Valadão GES, Viana PRM, Araújo AC. Introdução ao tratamento de Minérios. Belo Horizonte: Editora UFMG; 2004.

Recebido em: 09 Abr. 2019

Aceito em: II Mai. 2019 